\title{
Psychometric evaluation of the perceived access to health care questionnaire
}

\author{
Sara-Sadat Hoseini-Esfidarjani ${ }^{1}$ (D), Reza Negarandeh ${ }^{1,2^{*}}$ (D), Farzaneh Delavar ${ }^{1}$ and Leila Janani ${ }^{3}$ (D)
}

\begin{abstract}
Background and objective: Access to health care is a universal concern. Therefore, this study was conducted to develop a questionnaire to assess the Perceived Access to Health care based on Penchansky and Thomas's definition of access and the assessment of its psychometric properties.

Method: The initial questionnaire contains 31 items developed based on a deductive approach with an extensive review of the related literature. Content validity, face validity, construct validity, internal consistency, and instrument reliability were further examined. Data analysis was conducted using SPSS software version 24, R software version 4, and lavaan package.
\end{abstract}

Results: The initial questionnaire was examined using qualitative content validity, and the necessary modifications were applied to each item. The content validity ratio (CVR) was approved in 30 items with a value greater than 0.78 , and one item with a CVR value lower than 0.78 was removed. In the case of the content validity index (CVI), 29 items were approved with a CVI value of greater than 0.79 , and one item with a CVI value between 0.70 and 0.79 was revised. In qualitative face validity, all items were approved by a panel of experts and the participants. All 30 items with an impact score index higher than 1.5 were approved for the next steps. The confirmatory factor analysis results showed that the six-factor model of access to health care has an appropriate fit. Cronbach's alpha coefficient for the questionnaire was calculated 0.86. The value of Cronbach's alpha for the dimensions of availability, accessibility, affordability, accommodation, acceptability, and awareness were $0.61,0.76,0.66,0.60,0.80$, and 0.76 , respectively. The Intraclass Correlation Index (ICC) value for reliability (test-retest) of the whole instrument was calculated 0.94 using the two-way mixed absolute agreement method.

Conclusion: The success of health programs depends on eliminating barriers to access to provided health care services. One of the most critical barriers to understanding access is a perception of limited access. This questionnaire might be used further to understand perceived health care access in different global contexts.

Keywords: Validation study, Perception, Health care services, Health care services access, Factor analysis

\footnotetext{
* Correspondence: rnegarandeh@tums.ac.ir

${ }^{1}$ Nursing and Midwifery Care Research Center, Tehran University of Medical

Sciences, Nosrat St., Tohid Sq, Tehran 1419733171, Iran

${ }^{2}$ Department of Community Health \& Geriatric Nursing, School of Nursing

and Midwifery, Tehran University of Medical Sciences, Tehran, Iran

Full list of author information is available at the end of the article
}

(c) The Author(s). 2021, corrected publication 2022. Open Access This article is licensed under a Creative Commons Attribution 4.0 International License, which permits use, sharing, adaptation, distribution and reproduction in any medium or format, as long as you give appropriate credit to the original author(s) and the source, provide a link to the Creative Commons licence, and indicate if changes were made. The images or other third party material in this article are included in the article's Creative Commons licence, unless indicated otherwise in a credit line to the material. If material is not included in the article's Creative Commons licence and your intended use is not permitted by statutory regulation or exceeds the permitted use, you will need to obtain permission directly from the copyright holder. To view a copy of this licence, visit http://creativecommons.org/ licenses/by/4.0/. The Creative Commons Public Domain Dedication waiver (http://creativecommons.org/publicdomain/zero/1. 0/) applies to the data made available in this article, unless otherwise stated in a credit line to the data. 


\section{Introduction}

Healthcare access is a complex and global issue recognized as a fundamental human right [1]. Access to health care services plays a crucial role in the healthcare system's efficiency worldwide [2]. Hence, access is considered one of the main public policy issues in setting priorities or evaluating the healthcare system's performance [3]. Equality in health requires access to highquality health care services for all individuals and groups of people. Therefore, facilitating and maintaining individuals' access to essential health care services is one of the most important strategies to pursue to achieve social justice in each country's healthcare system [4].

A minimum of 400 million people worldwide is currently deprived of access to basic health care services. In addition, one out of every five people in the world lives in areas that are experiencing humanitarian crises [5]. Access problems, especially in low-income countries, actually threaten the achievement of the goals that the United Nations set in the Millennium Development Goals [6].

Evidence suggests that horizontal justice or equal access to equal needs in developed countries is also different for many care types [7]. Differences in access to health insurance between different races and individuals cause the death of more than 100,000 people each year [8]. Minorities often do not have access to health care services in a timely manner. The barriers to access to health care services have caused most minorities to experience delayed access to health care services or be ignored altogether. Such conditions lead to an increase in the cost of health care services, so that the progress in health care over the past 30 years has not shown a significant improvement in the rate of mortality among the minorities [9]. Shortage of services, distance from services, and inappropriate distribution of health care providers have limited access to health care services for rural residents [10]. Some Europeans still feel they are unable to access healthcare; in some European countries such as Bulgaria, Croatia, Latvia, Poland, Romania, and Sweden, reports suggest that more than $10 \%$ of the population do not access health care services [7].

Historically, the concept of health care services access has had different definitions that correspond to each health system's structural values. Most of the access models and definitions are based on market logic and meeting the needs; however, only very few models and definitions consider health as a basic human right [11].

Since the early 1970s, there has been extensive debate about the concept of health care access that still remains confusing [12]. Some researchers have used this term to denote the use of health care services. In contrast, other researchers use this term to convey a broad and multidimensional concept that describes the health system's characteristics [3]. Through analyzing this concept, Penchansky and Thomas (1981) have defined five dimensions for access, including 1) availability, 2) accessibility, 3) affordability, 4) accommodation, and 5) acceptability [13].

The first two dimensions are spatial in nature. Availability refers to the total number of services that the consumer can choose to receive. The accessibility dimension refers to the commuting time and the distance between the consumer's residence and the service center. The next three dimensions are non-spatial and belong to cost, service quality, and cultural factors [14]. For further explanation, it can be said that the availability dimension refers to the physical availability of health care resources with sufficient capacity to provide services [2]. Finally, the accessibility dimension refers to the location of health care delivery and the patient's residence, which includes concepts such as commuting time and distance [13].

The financial dimension shows the economic capacity of people to use the appropriate services [2]; in other words, this dimension indicates the relationship between the costs of health care providers and health insurance, the patients' ability to pay the prices, and their income [13]. The accommodation dimension is related to how the services are organized [15] and the provision of services based on the patients' needs and expectations. Finally, the acceptability dimension is related to the patients' and health care providers' personality traits and the impact of these traits on their attitudes and behaviors [13].

In 2015, Saurman added another dimension, which was called "awareness", to the model proposed by Penchansky and Thomas. By definition, awareness is the identification of the need for health care services. It is essential to know who the target of the services is, what they do if they are available, where and how they are used, and why they are used. Awareness also includes health literacy as another component of this dimension [1].

It can be said that access and use are not synonymous [16]. Access refers to the ability to receive the appropriate care from a proper healthcare provider, at the right time and place, depending on the context [1]. The decision to use available health care services depends on individuals' perception of the services [17]. In general, recognizing the perception of access to care for those in need is the first important step in reducing barriers to access [18]. Consumers' perception of access to health care services is crucial [10] because the decision to use available health care services depends on individuals' perception of the service and its reasonable costs [17]. Individuals' perceptions and judgments are often created by assessing the factors that make their traditions and 
culture important. These perceptions derived from their knowledge, values, and attitudes affect the place, time, and even whether patients should seek health care services or not [10]. To achieve public health, individuals' perception of health care services is essential for all the stakeholders in order to be able to provide successful interventions [17]. Perception enables judgment, and perception of access to health care services is, in fact, an alternative to demonstrating access to health care services that examines access management in a more straightforward way [11].

While there are specific indicators to measure different access dimensions separately, there is no extended indicator available. For example, health care services' availability has traditionally used indicators per capita of medical staff and resources such as hospital beds and medical technology units. Although these indicators show some of the available resources, they do not demonstrate anything about the quality of these resources or whether people should use them or not. Newer indicators of service availability such as distance, commuting time and cost, or public transportation to the nearest medical facilities can pose other methodological problems. These data underestimate the patients' actual communication because the closest service providers are not always the individuals' choice. In addition, the characteristics of each health system may widely vary across countries; given the development of disease management programs in many countries, measuring access to health care services will become increasingly difficult [19]. The literature review showed that many studies did not have appropriate and standard instruments to measure individuals' perception of healthcare services access $[7,17,20]$.

In Quinn's study, the dimensions of access in existing questionnaires have been investigated. From this study, it can be understood that each of the existing questionnaires focused on a specific dimension of access, and the available questionnaires did not examine all dimensions comprehensively and simultaneously [21]. Also, a study was observed on the development and validation of instruments for the perception of access to health care services [3], which did not cover all the dimensions of the present study; Besides, the present study applied a more robust method to analyze experimental data. Given the importance of individuals' perception of their access to health care services and the need to develop standard instruments to measure it, this study was conducted to develop and evaluate psychometric properties of the questionnaire to assess perceived access to health care services among the adults.

\section{Method}

\section{Designing of the questionnaire}

The initial version of the Perception of Access to Health care Services Questionnaire was developed based on the deductive method with a wide range of contents, definitions, and features of the concept of access as well as the perception of access and related questionnaires. The initial questionnaire was developed according to the five dimensions, which were introduced in Penchansky and Thomas' model of access (1981), and the sixth dimension, which was introduced in Saurman's (2015) study [1]. Thus, the proposed questionnaire includes six dimensions: 1) availability, 2) accessibility, 3) affordability, 4) accommodation, 5) acceptability, and 6) awareness. In addition, the psychometric evaluation was conducted on the instrument's initial version with 31 items on a 5point Likert scale (strongly agree to strongly disagree).

\section{Content validity}

The content of the questionnaire was examined in both quantitative and qualitative ways. Ten expert panel members were selected through the convenience method for the qualitative assessment of content validity. The opinion provided by the panel of experts in the field of access to health services, research, and psychometrics was obtained in terms of grammar, use of appropriate items and phrases, proper location of questionnaire items, and scoring procedure. Moreover, the questionnaire was sent to 15 experts for the quantitative analysis of the content validity, and the content validity ratio (CVR) and content validity index (CVI) were determined. In order to calculate CVR and after explaining the objectives of the questionnaire and providing the operational definitions related to the content of the questions, the experts were asked to rate each question based on a threepoint Likert scale, "The item is necessary", "The item is useful but unnecessary", and "The item is unnecessary". Then, the content validity ratio (CVR) was calculated using the formula. ${ }^{1}$ In examining the Content Validity Index (CVI), the experts were asked to determine the degree of relatedness based on a four-point scale: 1) not relevant, 2) somewhat relevant, 3) quite relevant, 4) highly relevant. The number of experts who chose options 3 and 4 was divided by the total number of experts afterward.

\section{Face validity}

In order to determine the face validity using the quantitative method, the items in the developed instrument were measured based on a five-point Likert scale quite important [5], important [4], moderately important [3], slightly important [2], and unimportant [1]. In this regard, the proposed questionnaire was provided to a panel of 20 experts. Thus, the ratio of participants who had chosen 4 and 5 for each item

${ }^{1} C V R=\frac{\text { ne }-N / 2}{N / 2}$ 
(frequency), as well as the sum of scores assigned to each item and the average score of each item (importance) were determined. Then, using the formula, ${ }^{2}$ the Impact score of each item was calculated. An Impact score equal to or greater than 1.5 was considered acceptable for each item. On the other hand, based on purposeful sampling, ten members of the panel of experts (written and email) and 30 members of the target group (face to face) were selected to conduct qualitative face validity. They were asked to comment on the level of item difficulty, comprehensibility of the phrases and the words, the appropriateness and relatedness of the items, as well as the ambiguity and misinterpretation of the words and phrases.

\section{Construct validity}

The construct validity determines the extent to which observational variables (items) can explain the study's main concept (the concept of perception of access to health care services). In the construct validity stage, the statistical population included patients referred to healthcare centers in the south of Tehran who were selected based on multi-stage sampling. Therefore, in the beginning, two areas were randomly selected from all the areas ( 5 areas) covered by the South Tehran Health Center. Then, two health centers were randomly selected from the health centers of these two areas. After that, the required samples were selected consecutively from those referred to these two health care centers. Inclusion criteria for the present study include the ability to speak in Persian, no apparent cognitive impairment, no history of specific diseases (dialysis, hemophilia, multiple sclerosis, and renal transplantation), citizenship and residence of Iran, recipients of services from health centers, and the age range between 20 and 60 years. According to the study of Myers et al., 300 people were considered as the sample size at this stage [22]. The objectives of the research were explained to all the participants before collecting data. They were then reassured about the confidentiality of the information, and informed consent was obtained.

The questionnaires were also completed through interviews with the participants from November 2018 to June 2019. The construct validity was determined using confirmatory factor analysis. Fit indices were used in the confirmatory factor analysis with experimental data. Model fit was considered good if the Tucker Lewis Index (TLI) [23], Normed-Fit Index (NFI), Non-Normed-Fit Index (NNFI), Goodness of Fit (GFI) were greater than or equal to 0.95. Comparative Fit Index (CFI) and Adjusted Goodness of Fit (AGFI) were greater than or

\footnotetext{
${ }^{2}$ impact score (frequency $\times$ Importance)
}

equal to 0.90 [24]. Root mean square error of approximation (RMSEA) was less than 0.05 . The ratio of $\chi^{2}$ to degrees of freedom ( $\left.\chi^{2} / \mathrm{df}\right)$ was below 2 [23].

\section{Reliability}

Reliability refers to the degree to which individuals maintain their position in a sample of repetitive dimensions. To assess it, the Intraclass Correlation Index (ICC) value was measured using the two-week test-retest approach for a group of 10 people from the Southern Health Centers [25]. In this study, the minimum ICC rate was considered 0.75 [26].

Internal consistency indicates whether the items in an instrument are conceptually compatible or not. Internal consistency was assessed using Cronbach's alpha. Besides, the internal correlation of the final questionnaire was examined, with 300 patients referred to the selected health centers. In this study, Cronbach's alpha of greater than 0.6 was considered acceptable $[27,28]$.

\section{Data analysis}

Mean (standard deviation) was used to describe quantitative variables, and frequency report (percentage) was used to describe categorical variables. These analyzes were performed in SPSS software version 24. Also, $R$ software version 4 and lavaan package were used for confirmatory factor analysis. Substitution of the variable's mean for the missing data points on that variable was used for the missing data. In addition, fit indices were used in the confirmatory factor analysis with experimental data.

\section{Findings}

In qualitative content analysis, the questionnaire was reviewed, and the necessary corrections were applied to each item based on the opinions obtained from the panel of experts. It should also be noted that only 9 of the 15 experts invited to the panel responded. Therefore, according to the numbers provided by the Lawshe table, the items with a CVR coefficient of greater than 0.78 were considered acceptable, and their validity was confirmed. Accordingly, 30 items with a CVR value of greater than 0.78 were confirmed in the present study. However, one item with a CVR value of lower than 0.78 was removed. Also, the content validity index (CVI) was evaluated for 31 items. In the case of CVI, the items with a score higher than 0.79 were considered appropriate, the items with a score between 0.70 and 0.79 were considered as the items with the need to be revised, and the items with a score equal to and lower than 0.70 were considered as unacceptable items and were then removed. Accordingly, 29 items with a content validity index value of greater than 0.79 were confirmed. A single item with a score of between 0.70 and 0.79 was 
revised. The item "When providing education, the underlying factors, as well as my ability to understand health information, are taken into account." in the dimension of "awareness" was changed to "My living conditions are taken into accounts, such as marital status, ability to pay, and cultural differences". The item "I recognize the need for specialized and sub-specialized services" in the dimension of Affordability with a score of lower than 0.70 was removed. In fact, the same item was removed in both CVI and CVR.

As a result, the remaining 30 (see technical appendix in Additional file 1) items in the content validity analysis were examined for face validity. Findings from the qualitative face validity showed that the panel of experts and participants approved the level of difficulty, the degree of appropriateness and ambiguity of the scale, and the 5point Likert scale. In the quantitative face validity analysis, the item effect size was calculated, and all items were retained for the next steps since the obtained score for each of the 30 items was higher than 1.5.

Thus, the final questionnaire with 30 items and six constructs was completed through interviews with 300 individuals referred to the research setting. Finally, the data for 276 individuals were analyzed because some data within 24 questionnaires were incomplete. The participants aged between 20 and 60 years with an average of 37.8 years and a standard deviation of 10.27 years.

Table 1 shows the distribution of the participants' demographic characteristics (sex, level of education, place of employment, insurance status, and supplementary insurance).

The confirmatory factor analysis results showed that the six-factor model of perception of access to health care services in patients referred to health centers provides a good fit. GFI, AGFI, TLI, NNFI, NFI, CFI, RMSEA, and $X^{2} / \mathrm{df}$ were calculated; the indicators confirm the model fit and acceptable values given in Table 2. Figure 1 shows the factor loading of latent factors.

Table 3 shows the internal consistency of the Perception of Access to Health care services Questionnaire using Cronbach's alpha. It is reported that the Cronbach's alpha for the subscales of the Perception of Access to Health care services questionnaire was calculated between 0.60 and 0.80 . While the value of Cronbach's alphafor three dimensions was (0.6-0.7) indicates an acceptable level of reliability [28], the rest have good, with The value of Cronbach's alpha greater than 0.7. However, the obtained Cronbach's alpha for the whole questionnaire was 0.86. Besides, the questionnaire's obtained ICC value was 0.94 (CI95\%: 0.78, 0.98), which was calculated using the two-way mixed absolute agreement method.

\section{Discussion}

Access to health care is an important concept in public health [29]. Access to health care can be defined in many ways. Some definitions address whether people actually use such services or not and define their use of services as access. Other definitions focus on health

Table 1 Demographic characteristics of the individuals referred to healthcare centers $(n=276)$

\begin{tabular}{|c|c|c|c|}
\hline \multirow[t]{2}{*}{ Demographic characteristics } & & \multicolumn{2}{|c|}{ Frequency } \\
\hline & & Number & Percentage \\
\hline \multirow[t]{3}{*}{ Sex } & Female & 212 & 76.9 \\
\hline & Male & 60 & 21.7 \\
\hline & Unanswered & 4 & 1.4 \\
\hline \multirow[t]{5}{*}{ Educational level } & Illiterate & 11 & 4 \\
\hline & Elementary or Middle school & 79 & 28.6 \\
\hline & High-school diploma & 97 & 35.2 \\
\hline & Graduated & 58 & 30.8 \\
\hline & Unanswered & 4 & 1.4 \\
\hline \multirow[t]{3}{*}{ Occupation } & Employed & 95 & 34.4 \\
\hline & Unemployed & 179 & 64.9 \\
\hline & Unanswered & 2 & 0.7 \\
\hline \multirow[t]{3}{*}{ Insurance status } & Supported & 224 & 81.2 \\
\hline & Unsupported & 46 & 16.7 \\
\hline & Unanswered & 6 & 2.1 \\
\hline \multirow[t]{3}{*}{ Complementary insurance status } & Supported & 57 & 20.7 \\
\hline & Unsupported & 213 & 77.2 \\
\hline & Unanswered & 6 & 2.1 \\
\hline
\end{tabular}


Table 2 Fit indices for confirmatory factor analysis model $(n=276)$

\begin{tabular}{|c|c|c|c|c|c|c|c|c|}
\hline Model fit indices & $x^{2 / d f}$ & RMSEA & GFI & AGFI & NNFI & TLI & NFI & CFI \\
\hline Confirmatory factor analysis & 1.147 & 0.024 & 0.938 & 0.926 & 0.981 & 0.981 & 0.884 & 0.983 \\
\hline Thresholds for acceptable fit & $<5$ & $<0.08$ & $>0.8$ & $>0.85$ & $>0.80$ & $>0.80$ & $>0.80$ & $>0.85$ \\
\hline Thresholds for good fit & $<2$ & $<0.05$ & $>0.95$ & $>0.90$ & $>0.95$ & $>0.95$ & $>0.95$ & $>0.90$ \\
\hline
\end{tabular}

insurance coverage or eligibility to receive health care in the event of illness. Some other definitions highlight the possibility that someone can access health care services if needed [30]. In general, access to health care services is traditionally measured using population-level parameters such as the availability, distribution, and proximity of health facilities to the population. These approaches do not consider important factors such as individuals' attitudes, perceptions, expectations, experiences, and socio-cultural factors such as norms and belief systems that affect people. In contrast, these factors can enable or hinder access to health care [29].

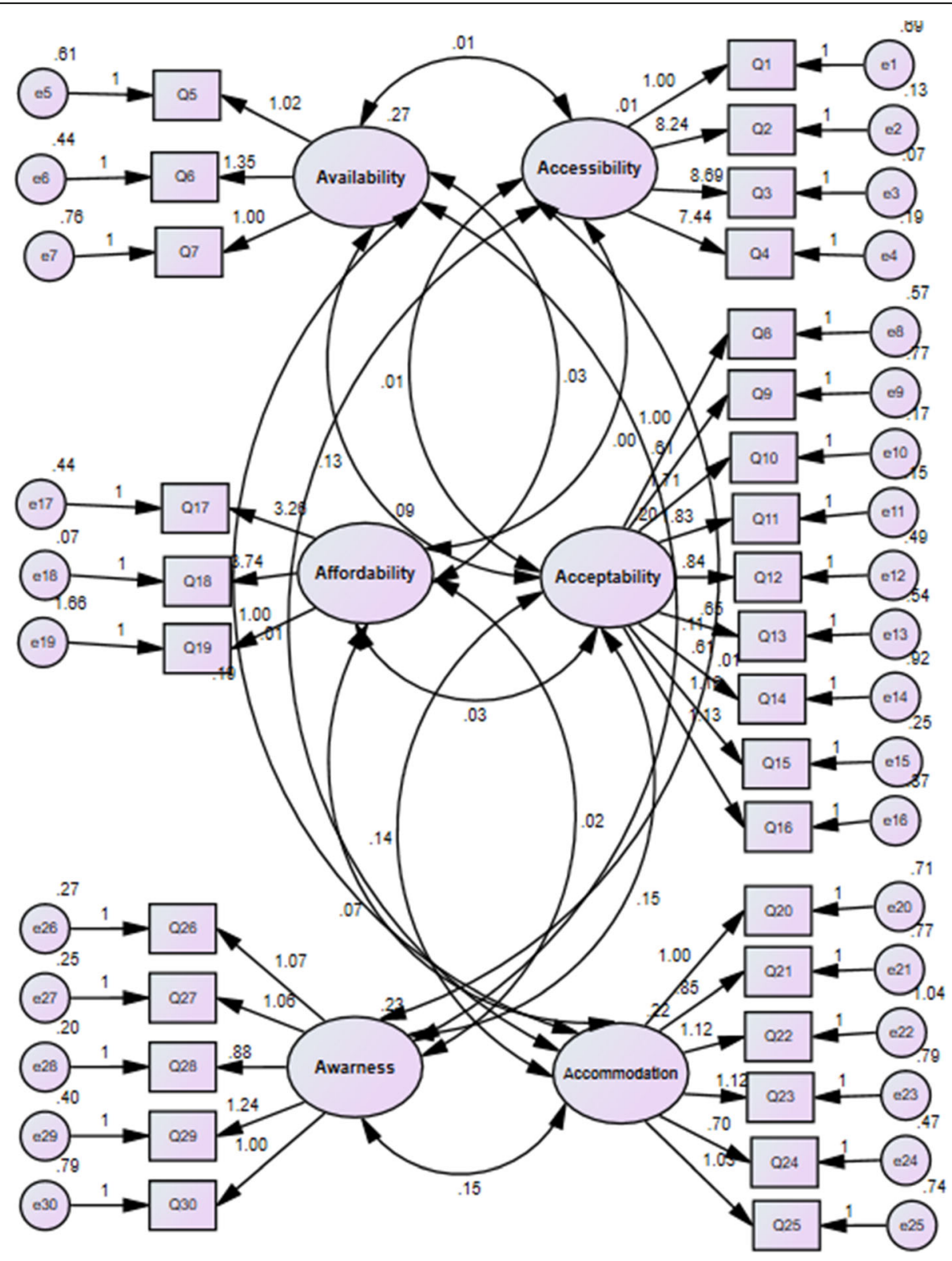

Fig. 1 Structural Equation Modeling 
Table 3 The Cronbach's alpha coefficient for the Perception of Access to Health care services Questionnaire

\begin{tabular}{lll}
\hline Dimensions & The number of items & $\begin{array}{l}\text { Reliability } \\
\text { (Cronbach's alpha coefficient) }\end{array}$ \\
\hline Availability & 3 & 0.61 \\
Accessibility & 4 & 0.76 \\
Affordability & 3 & 0.66 \\
Accommodation & 6 & 0.60 \\
Acceptability & 9 & 0.80 \\
Awareness & 5 & 0.76 \\
Perception of Access to Health care services & 30 & 0.86 \\
\hline
\end{tabular}

Evaluation of the success of executive programs in access to health care services requires a valid measure of the perception of access to health care services; the questionnaire that was developed and underwent psychometric evaluation in this study could help achieve such measurement.

Some studies have been conducted on the perception of access to health care services [7, 17, 31, 32]. However, none of them used a valid and well-known instrument. These studies have only examined one or more access dimensions that could not cover all the present study areas. Pyne et al. (2020) developed an instrument to measure the perception of access to mental health care services; nonetheless, this study did not aim to validate the instrument [33]. Zandom et al. (2017) developed a scale to measure perceptions of access to health care services among the disabled and their non-disabled counterparts in Nigeria. This instrument included 25 items and was developed based on the theories proposed by Levesque et al. (2013), Peters et al. (2008), Obrist et al. (2007), and Penchasky \& Thomas (1981) [3].

It can be claimed that the questionnaire which was developed in the present study is distinct because it is based on the modified Penchansky and Thomas's Theory of Access [1]. Their model provides a classified definition of access and summarizes a set of specific dimensions that describe the fit between the health care system and the general population. All dimensions of access have equal values in this theory, just like chain knots [34].

Accordingly, the questionnaire for perception of access to health care services was developed in six subscales and 31 items in the present study: 1) availability which refers to the adequacy of supply and examines the available services and resources to meet the potential and actual needs of the consumers and communities; 2) accessibility which evaluates the available service in accordance with the reasonable proximity to the consumer in terms of time and distance; 3 ) affordability which refers to the direct costs to the health care providers and consumers; 4) accommodation which refers to the adequacy of classification of services in order to be accepted and used by the consumers; 5) acceptability which highlights the provider and consumers' attitudes about the characteristics of the service as well as their social or cultural concerns; and 6) awareness represents the concept that services can help maintain awareness through effective communication and information strategies with the target users (physicians, patients, and the general public), including attention to health context and literacy [1].

In both the quantitative and qualitative approaches to content validity, only one item was removed, while all the items were retained in the analysis of face validity. Thus, the questionnaire for the perception of access to health care services was observed for construct validity with six subscales and 30 items. In addition, confirmatory factor analysis was used to evaluate the construct validity of the Perceptions of Access to Health care services. The desired values of fit indicators in confirmatory factor analysis confirmed the construct validity of the Questionnaire and indicated the proper classification of the questions. A good questionnaire is generally supported by a reasonable theoretical basis so that the items can operationally define the targeted questionnaire $[35,36]$.

For this reason, confirmatory factor analysis (CFA) has been used in the present study. Moreover, CFA is used when there is a strong model assumption. CFA aims to investigate the existence of a previously confirmed construct with a new dataset [37] However, in the study of Zandom et al.; The construct validity showed that the items of the questionnaire were related to the sixdimensional hypothetical structure based on models of access to health services [3].

Cronbach's alpha was calculated to estimate the total instrument's internal consistency and its different dimensions; the obtained Cronbach's alpha for the entire questionnaire was 0.86 and for the dimensions were between 0.6 and 0.8 . Besides, there are several reports of acceptable alpha values ranging from 0.70 to 0.95 . A seminal study by Tavakol and Dennick noted that if Cronbach's alpha is too large, it may indicate redundant items that raise similar questions with only different 
phrases. Therefore, the maximum alpha value is recommended to be 0.90 [38]. Although the whole instrument's internal consistency and its three dimensions (awareness, acceptability, and acceptability) were good, the other three dimensions (availability, affordability, and accommodation) of the questionnaire had acceptable Cronbach's alpha. Tavakol and Dennick (2011) wrote that "A low value of alpha could be due to a low number of questions, poor interrelatedness between items or heterogeneous constructs" (p.54) [38].

In this study, the dimensions of availability and affordability in the perceived access to health care questionnaire had only three items that could explain the low Cronbach's alpha. Although the dimension of accommodation has six items, Cronbach's alpha in this dimension was also low, which could be due to the cultural differences of the study participants. Jih-Yuan Chen's study suggests that low Cronbach's alpha levels may be due to cultural bias [39]. Tehran (research setting) embraces people with different cultures. It may be justified that understanding people with different cultural backgrounds has caused them to answer the following questions in different ways. Also, there are broader concepts in this dimension than other dimensions that increase the possibility of heterogeneous items. This questionnaire is designed based on the modified Penchasky \& Thomas model. It seems that the development of the model in the future can provide more clarity in the definition of its dimensions and help revise the questionnaire items accordingly. Nevertheless, the dimensions with low Cronbach's alpha need to be further examined in future studies.

In addition, the Intra-Class Correlation Index (ICC) was calculated to determine the reliability of the instrument using the test-retest approach. ICC is a reliable indicator that reflects both the degree of correlation and the compatibility between the measurements; the obtained ICC value in the present study was equal to 0.94 . According to $\mathrm{Koo}$ and $\mathrm{Li}$, this rate indicates excellent reliability of the questionnaire [26].

It can be concluded that the "Perceived Access to Health care Questionnaire" was developed with 30 items; it has acceptable content validity, face validity, construct validity, and reliability. Therefore, this scale can be used in future studies to measure perceived access to health care services.

In some cases, perceptions about access to health services can be a barrier to using health services; we can identify these mental barriers and evaluate interventions' effectiveness by measuring them. The removal of such mental barriers to greater access to the services helps to achieve equity in access.

A limitation of this study is that item difficulty was not measured, and other types of validity, such as predictive and concurrent validity were not tested. Therefore, further testing is required to provide more evidence regarding the validity of this tool.

\section{Conclusion}

The success of national development programs largely depends on the health sector's success in achieving its objectives. The proper use of health care services in different places and among different groups of people is considered a prerequisite for this success [40]. In this case, the clients' perception of access to health care services is crucial because their perceptions affect when and where they should seek and receive health care services. In this study, the questionnaire on the perception of access to health care services was developed, and its psychometric properties were examined. Therefore, it is expected that related field research will be accelerated with the introduction of this questionnaire. By measuring perceived access to health care services, health policymakers and practitioners may detect and eliminate perceptual and actual obstacles to using health care services.

\section{Supplementary Information}

The online version contains supplementary material available at https://doi. org/10.1186/s12913-021-06655-2.

Additional file 1. Technical appendix.

\section{Acknowledgments}

This research has been supported by Tehran University of Medical Sciences \& Health Services grant (No: 38062-99-01-97). In addition, we thank Mr. Farshad Sharifi-Assistant Professor in Elderly Health Research Center, Endocrinology and Metabolism Population Sciences Institute, Tehran University of Medical Sciences- who participated in the supplementary analysis in this study.

Authors agreement statement

All authors confirm that the manuscript has been read and approved by all named authors and that there are no other persons who satisfied the criteria for authorship but are not listed.

\section{Authors' contributions}

S-S. H-E.: Conception \& design, literature search, drafting, and revising of manuscript. R. N.: Conception \& design, supervision of study, drafting and revising of manuscript. F.D.: Data acquisition, revising of manuscript. L.J.: Data analysis, revising of manuscript. The author(s) read and approved the final manuscript.

\section{Funding}

The authors received no specific funding for this work.

\section{Availability of data and materials}

The datasets analyzed during the current study are available from the corresponding author on reasonable request.

\section{Declarations}

Ethics approval and consent to participate

The study was evaluated and approved by the School of Nursing and Midwifery \& Rehabilitation-Tehran University of Medical Sciences (Ethical code: IR.TUMS.FNM.REC.1397.014) and followed the Declaration of Helsinki Ethical Principles for Medical Research Involving Human Subjects. Written 
informed consent was obtained from all individual participants included in the study and

We confirm that all experiments were performed in accordance with relevant guidelines and regulations.

\section{Consent for publication}

Not applicable.

Manuscript did not include details, images, or videos relating to an individual person.

\section{Competing interests}

The authors have declared that no competing interests exist.

\section{Author details}

${ }^{1}$ Nursing and Midwifery Care Research Center, Tehran University of Medical Sciences, Nosrat St., Tohid Sq, Tehran 1419733171, Iran. ${ }^{2}$ Department of Community Health \& Geriatric Nursing, School of Nursing and Midwifery, Tehran University of Medical Sciences, Tehran, Iran. ${ }^{3}$ Department of Biostatistics, School of Public Health, Iran University of Medical Sciences, Shahid Hemmat Highway, Tehran 1449614535, Iran.

\section{Received: 27 November 2020 Accepted: 17 June 2021}

\section{Published online: 02 July 2021}

\section{References}

1. Saurman E. Improving access: modifying Penchansky and Thomas's theory of access. J Health Serv Res Policy. 2016;21(1):36-9. https://doi.org/10.11 77/1355819615600001.

2. Levesque J-F, Harris Mark F, Grant R. Patient-centred access to health care: conceptualising access at the interface of health systems and populations. Int J Equity Health. 2013;12(1):18. https://doi.org/10.1186/1475-9276-12-18.

3. Zandam HU, Hanafiah JM, Hayati KS, et al. Development and validation of perceived access to health care measurement instrument. Int J Public Health Clin Sci. 2017;4(5):158-71. Retrieved from http://www.publichea Ithmy.org/ejournal/ojs2/index.php/ijphcs/article/view/494.

4. Negarandeh R, Haydeh N, Maryam HP. Evaluating the perception of martyrs' parents of access to the services of health monitoring plan provided by Foundation of Martyrs and Veterans Affair of 10 and 11 districts of Tehran in 2014. Nurs Pract Today. 2016;3(3):99-106. Retrieved from https://npt.tums.ac. ir/index.php/npt/article/view/165.

5. World Health Organization. Expanding access to health services with selfcare interventions. 2019. Retrieved from: https://www.who.int/ reproductivehealth/self-care-interventions/access-health-services/en/.

6. Eide $\mathrm{AH}$, Hasheem $\mathrm{M}$, Mustafa $\mathrm{K}$, et al. Perceived barriers for accessing health services among individuals with disability in four African countries. PLoS One. 2015;10(5):e0125915. https://doi.org/10.1371/journal. pone.0125915.

7. Cylus J, Irene P. An analysis of perceived access to health care in Europe: how universal is universal coverage? Health Policy. 2015;119(9):1133-44. https://doi.org/10.1016/j.healthpol.2015.07.004

8. Wang F. Measurement, optimization, and impact of health care accessibility: a methodological review. Ann Assoc Am Geogr. 2012;102(5):1104-12. https://doi.org/10.1080/00045608.2012.657146.

9. Mary Brown Walker. A phenomenological study on perceptions of health care access for African Americans and Latino Americans: University of Phoenix; 2015. retrieved from https://search.proquest.com/openview/1a3a 9625e12ff18eaca682c914da265e/1?pq-origsite=gscholar\&cbl=18750\&diss=y.

10. Terry D, Kaye E, Alan C, et al. Heterogeneity of rural consumer perceptions of health service access across four regions of Victoria. J Rural Soc Sci. 2017; 32(2):6. retrieved from https://egrove.olemiss.edu/jrss/vol32/iss2/6/.

11. Julián Alfredo Fernández-Niño, Chavarro Lud Magdy, Vásquez-Rodríguez Ana Beatriz, et al. Perception of effective access to health services in Territorial Spaces for Training and Reincorporation, one year after the peace accords in Colombia: a cross-sectional study. F1000Research. 2019:8:2140. https://doi.org/10.12688/f1000research.21375.2

12. Thiede M, Patricia A, Di Ml, et al. Exploring the dimensions of access. In: McIntyre D, Mooney G, editors. The economics of health equity. Cambridge: Cambridge University Press; 2007. p. 103-23. https://doi.org/10.1017/ CBO9780511544460.007.

13. Kyriopoulos I-I, Dimitris Z, Anastasis S, et al. Barriers in access to healthcare services for chronic patients in times of austerity: an empirical approach in
Greece. Int J Equity Health. 2014;13(1):54. https://doi.org/10.1186/1475-92 76-13-54.

14. Shrestha J. Evaluation of access to primary healthcare: a case study of Yogyakarta, Indonesia: [MSc thesis on the internet] University of Twente Faculty of geo-information and earth observation (ITC); 2010.

15. Jeannie L. Haggerty, \& Levesque Jean-Frédéric. Validation of a new measure of availability and accommodation of health care that is valid for rural and urban contexts. Health Expect. 2017;20(2):321-34. https://doi.org/10.1111/ hex.12461.

16. Bath B, Megan J, Darren M, et al. Factors associated with reduced perceived access to physiotherapy services among people with low back disorders. Physiother Can. 2016;68(3):260-6. https://doi.org/10.3138/ptc.2015-50.

17. Onyeneho NG, Amazigo Uche V, Njepuome Ngozi A, et al. Perception and utilization of public health services in Southeast Nigeria: implication for health care in communities with different degrees of urbanization. Int J Equity Health. 2016;15(1):12. https://doi.org/10.1186/s12939-016-0294-z.

18. Weber M, Lisa T, Schmiege Sarah J, et al. Perception of access to health care by homeless individuals seeking services at a day shelter. Arch Psychiatr Nurs. 2013;27(4):179-84. https://doi.org/10.1016/j.apnu.2013.05.001.

19. Allin S, Cristina M, Corinna S, et al. Measuring inequalities in access to health care: a review of the indices. Belgium: European Commission Brussels; 2007. retrieved from: https://scholar.google.com/scholar?hl=en\&as_sdt=0\%2 $\mathrm{C} 5 \& \mathrm{q}=\% 22$ Measuring + inequalities+in+access+to+health+care.+A+review + oftthe+indices $\% 22 \& b t n G$.

20. Fitzpatrick AL, Powe Neil R, Cooper Lawton S, et al. Barriers to health care access among the elderly and who perceives them. Am J Public Health. 2004;94(10):1788-94. https://doi.org/10.2105/ajph.94.10.1788.

21. Quinn M, Claire R, Jane F, et al. Survey instruments to assess patient experiences with access and coordination across healthcare settings: available and needed measures. Med Care. 2017;55(Suppl 7 1):S84.

22. Myers ND, Soyeon A, Ying J. Sample size and power estimates for a confirmatory factor analytic model in exercise and sport: a Monte Carlo approach. Res Q Exerc Sport. 2011;82(3):412-23. retrieved from:. https://doi. org/10.1080/02701367.2011.10599773.

23. Shi G, Jun W, Xu X, et al. Culture-related grief beliefs of Chinese Shidu parents: development and psychometric properties of a new scale. Eur J Psychotraumatol. 2019;10(1):1626075. https://doi.org/10.1080/20008198.201 9.1626075.

24. S Parry. Fit indices commonly reported for CFA and SEM. Cornell Statistical Unit. 2020. retrieved from: https://www.cscu.cornell.edu/news...

25. Mohamad Adam Bujang, \& Baharum Nurakmal. A simplified guide to determination of sample size requirements for estimating the value of intraclass correlation coefficient: a review. Arch Orofac Sci. 2017;12(1). retrieved from: https://scholar.google.com/scholar?hl=en\&as_sdt=0\%2 $C 5 \& q=\% 22 \mathrm{~A}+$ simplified+guide+to+determination+of+sample+size+ requirements+for+estimating+the+value+of+intraclass+correlation+ coefficient $\% 3 \mathrm{~A}+\mathrm{a}+$ review\% $22 \& \mathrm{btnG}=$

26. Koo TK, Li Mae Y. A guideline of selecting and reporting intraclass correlation coefficients for reliability research. J Chiropract Med. 2016;15(2): 155-63. https://doi.org/10.1016/j.jcm.2016.02.012.

27. Daud KAM, Zulkarnaen KN, Rasdan IA, et al. Validity and reliability of instrument to measure social media skills among small and medium entrepreneurs at Pengkalan Datu River. Int J Dev Sustain. 2018;7(3): 1026-37.

28. Martin CR, Emily S-MG. A 'good practice'guide for the reporting of design and analysis for psychometric evaluation. J Reprod Infant Psychol. 2013; 31(5):449-55.

29. Yakob B, Purity NB. Correlates of perceived access and implications for health system strengthening-lessons from HIV/AIDS treatment and care services in Ethiopia. PLoS One. 2016;11(8):e0161553. https://doi.org/10.1371/ journal.pone.0161553.

30. William D Savedoff. A moving target: universal access to healthcare services in Latin America and the Caribbean. Working Paper; 2009. https://www. econstor.eu/handle/10419/51524.

31. Bezem J, Debbie H, Ria R, et al. Improving access to school health services as perceived by school professionals. BMC Health Serv Res. 2017;17(1):743. https://doi.org/10.1186/s12913-017-2711-4.

32. Tanner EC, Vann Richard J, Elvira K. Consumer-level perceived access to health services and its effects on vulnerability and health outcomes. J Public Policy Mark. 2020;39(2):240-55. https://doi.org/10.1177/074391562 0903299. 
33. Pyne JM, Adam KP, Fischer Ellen P, et al. Development of the Perceived Access Inventory: A patient-centered measure of access to mental health care. Psychol Serv. 2020;17(1):13. https://doi.org/10.1037/ser0000235.

34. Otieno PO, Achach WEO, Mohamed Shukri M, et al. Access to primary healthcare services and associated factors in urban slums in Nairobi-Kenya. BMC Public Health. 2020;20:981. https://doi.org/10.1186/s12889-020-09106-5.

35. Gendall P. A framework for questionnaire design: Labaw revisited. Marketing bulletin-department of marketing massey university. 1998;9:28-39. https:// scholar.google.com/scholar?hl=en\&as_sdt=0\%2C5\&q=\%22A+Framework+ for+Questionnaire+Design\%3A+Labaw+Revisited\%22\&btnG

36. Jenn NC. Designing a questionnaire. Malaysian Fam Phys. 2006;1 (1):32. https://www.ncbi.nlm.nih.gov/pmc/articles/PMC4797036/.

37. Orcan F. Exploratory and confirmatory factor analysis: which one to use first. J Measurement Eval Educ Psychol. 2018;9(4):414-21. retrieved from: https:// scholar.google.com/scholar?hl=en\&as $s \mathrm{dt}=0 \% 2 \mathrm{C} 5 \mathrm{qq}=\% 22$ Exploratory+and + Confirmatory+Factor+Analysis\%3A+Which+One+to+Use+First\%3F\%22 \&btnG.

38. Tavakol M, Reg D. Making sense of Cronbach's alpha. Int J Med Educ. 2011; 2:53-5. https://doi.org/10.5116/ijme.4dfb.8dfd.

39. Jih-Yuan Chen. Stimulate reflection and foster critical thinking in nursing education. Int J Curr Res. 2017;9(7):55089-55093. retrived from: http://www. journalcra.com.

40. Mehdi H, Rajabi G. Health Care Services Utilization in Iran. Iran J Public Health. 2017;46(6):863-4. retrieved from: https://scholar.google.com/schola $r ? h l=e n \& a s \_s d t=0 \% 2 C 5 \& q=\% 22 \mathrm{Health}+$ Care + Services+Utilization+in+lra $n \% 22 \& b \operatorname{tnG}=$

\section{Publisher's Note}

Springer Nature remains neutral with regard to jurisdictional claims in published maps and institutional affiliations.

Ready to submit your research? Choose BMC and benefit from:

- fast, convenient online submission

- thorough peer review by experienced researchers in your field

- rapid publication on acceptance

- support for research data, including large and complex data types

- gold Open Access which fosters wider collaboration and increased citations

- maximum visibility for your research: over $100 \mathrm{M}$ website views per year

At $\mathrm{BMC}$, research is always in progress.

Learn more biomedcentral.com/submissions 